\title{
Hyperdiploid B Acute Lymphoblastic Leukemia
}

National Cancer Institute

\section{Source}

National Cancer Institute. Hyperdiploid B Acute Lymphoblastic Leukemia. NCI Thesaurus. Code C80344.

A B-cell acute leukemia characterized by the presence of lymphoblasts which contain more than 50 and usually less than 66 chromosomes. It is commonly seen in children and rarely in adults. It has a favorable clinical outcome. 